\title{
Ego-vehicle speed estimation on spectral analysis using fast chirp modulation radar
}

\author{
Keiji Jimi $^{1, \text { a) }}$, Ryohei Nakamura ${ }^{2}$, and Takeki Ogitsu ${ }^{1}$ \\ ${ }^{1}$ Center for Research on Adoption of NextGen Transportation Systems, \\ Gunma University, Maebashi-shi, Gunma, 371-0044 Japan \\ ${ }^{2}$ Graduate School of Science and Engineering, National Defense Academy of Japan, \\ Hashirimizu, Yokosuka, Kanagawa 239-8686, Japan
}

a)kei.jimi@gunma-u.ac.jp

Abstract: A method for ego-vehicle speed estimation using a fast chirp modulation (FCM) radar is proposed, which estimates the vehicle speed spectrum using a discrete Fourier transform and tracks the peak of the spectrum to estimate vehicle speed. Because it is applied to the intermediate-frequency signal, it can estimate vehicle speed without detecting objects. The proposed method can estimate low speeds which cannot be measured by wheel speed sensors. The estimated results of experiments using a vehicle were compared with those of in-vehicle sensors, revealing that the method can estimate vehicle speed using an FCM radar with low antenna elements.

Keywords: FCM radar, Doppler, vehicle speed, traveled distance, 2D discrete Fourier transform

Classification: Sensing

\section{References}

[1] K.T. Leung, J.F. Whidborne, D. Purdy, and P. Barber, "Road vehicle state estimation using low-cost GPS/INS," Mechanical System and Signal Processing, vol. 25, no. 6, pp. 1988-2004, Aug. 2011. DOI: 10.1016/j.ymssp.2010.08.003

[2] S. Danskin, P. Bettinger, and T. Jordan, "Multipath mitigation under forest canopies: a choke ring antenna solution," Forest Science, vol. 55, no. 2, pp. 109 116, April 2009. DOI: 10.1093/forestscience/55.2.109

[3] G. Grisetti, R. Kummerle, C. Stachniss, and W. Burgard, "A tutorial on graphbased SLAM,” IEEE Intell. Transp. Syst. Mag., vol. 2, no. 4, pp. 31-43, 2010. DOI: 10.1109/MITS.2010.939925

[4] R.W. Wolcott and R.M. Eustice, "Fast LIDAR localization using multiresolution Gaussian mixture maps," Proc. International Conf. on Robotics and Automation (ICRA), Seattle, Washington, USA, pp. 2814-2821, July 2015. DOI: 10.1109/ICRA.2015.7139582

[5] C. Häne, L. Heng, G.H. Lee, F. Fraundorfer, P. Furgale, T.Sattler, and M. Pollefeys, "3D visual perception for self-driving cars using a multi-camera system: calibration, mapping, localization, and obstacle detection," Image Vision Computing, vol. 68, pp. 14-27, Dec. 2017. DOI: 10.1016/j.imavis. 2017.07.003 
[6] K. Imou, T. Okamoto, Y. Kaizu, and H. Yoshii, "Ultrasonic Doppler speed sensor for autonomous vehicles," Journal of Japanese Society of Agricultural Machinery, vol. 63, no. 2, pp.39-46, 2001. DOI: 10.11357/jsam1937.63.2_39

[7] D. Lhomme-Desages, C. Grand, F. Ben Amar, and J.-C. Guinot, "Doppler based ground speed sensor fusion and slip control for a wheeled rover," IEEE/ASME Trans. Mechatronics, vol. 14, no. 4, pp. 484-492, Aug. 2009. DOI: 10.1109/ TMECH.2009.2013713

[8] T. Hague, J.A. Marchant, and N.D. Tillett, "Ground based sensing systems for autonomous agricultural vehicles," Computers and Electronics in Agriculture, vol. 25, no. 1-2, pp. 11-28, Jan. 2000. DOI: 10.1016/S0168-1699(99)00053-8

[9] D. Kellner, M. Barjenbruch, J. Klappstein, J. Dickmann, and K. Dietmayer, "Instantaneous ego-motion estimation using Doppler radar," Proc. $16^{\text {th }}$ International IEEE Conf. on Intelligent Transportation Systems (ITSC), The Hague, Netherlands, pp. 1-6, Oct. 2013. DOI: 10.1109/ITSC.2013.6728341

[10] C. Iovescu and S. Rao, "The fundamentals of millimeter wave sensors," Texas Instruments, https://www.ti.com/lit/wp/spyy005a/spyy005a.pdf?ts=16226126 29667\&ref_url=https\%253A\%252F\%252Fwww.google.com\%252F, accessed June 2, 2021.

\section{Introduction}

Localization of autonomous vehicles is important for safe driving. In-vehicle sensors for accurate localization have been developed. The global navigation satellite system (GNSS) determines the absolute position of a vehicle using satellite signals [1]. GNSS performance is degraded by multipath interference occurring in urban, forest, indoor, and other environments [2]. Relative positioning using external sensors has attracted attention to solve this problem. The relative position is estimated from observational data. There are two approaches to relative positioning: simultaneous localization and mapping (SLAM) and vehicle trajectory tracking.

In SLAM, observational data are matched with the landmarks on a map. An external sensor observes the shape of the object. The observational data of the features are compared with landmarks, and the relative position is estimated from the correlation between observational data and the landmarks. Accurate external sensors, such as LIDAR and image sensors, are used in SLAM because SLAM requires the accurate shape and position of objects $[3,4,5]$. However, these sensors tend to perform poorly in bad weather, such as rain, snow, and fog. SLAM performance is degraded in environments without landmarks, such as tunnels and mountains.

In the vehicle trajectory tracking method, the trajectory is measured by the integration of ego-motion estimated from observation data. Methods that do not utilize observational data have also been developed. Ego-vehicle speed can be estimated using a Doppler sensor $[6,7,8]$. A Doppler sensor pointed at the ground measures the Doppler effect from the ground surface, and the ego-vehicle speed is calculated from the relative speed and angle of the Doppler sensor. However, the Doppler sensor angle changes with the jolt of the vehicle. To reduce the effect of jolts, measurement using an in-vehicle radar for obstacle detection was proposed [9]. The in-vehicle radar detects the angle and relative speed of the objects as point data, and the ego-vehicle speed is estimated from the curved distribution of the point 
group. However, high-resolution radar is required to detect many accurate points.

Herein, an ego-vehicle speed estimation method involving spectral analysis using on-vehicle radar is proposed. The method uses fast chirp modulation (FCM) radar [10] and estimates a spectrum correlated with vehicle speed (VS) from the intermediate-frequency (IF) signal obtained from the FCM radar. The ego-vehicle speed is estimated by tracking the peak of the VS spectrum. The method can be applied to low-resolution FCM radar because it does not require object detection. An experiment using a vehicle was conducted to estimate the ego-vehicle speed detection and travel distance.

\section{Ego-vehicle speed estimation}

\subsection{Relationship to relative speed}

The ego-vehicle speed is estimated from the relative speed and direction of stationary objects. As shown in Fig. 1(a), a traveling vehicle measures the relative speed $v_{r}$ and direction $\theta$ of the object. The ego-vehicle speed is estimated as follows:

$$
\begin{aligned}
v_{r} & =v \cos \theta \\
v & =\frac{v_{r}}{\cos \theta} .
\end{aligned}
$$

The directional accuracy affects the estimation. In-vehicle radar has a wide beam to observe surrounding obstacles, and the directions of the objects change with traveling. The direction error is caused by the side lobes and multipath interference.

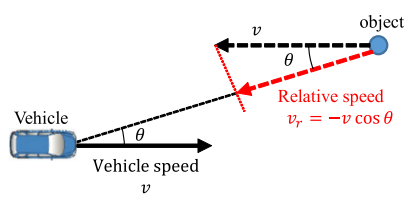

(a) vehicle speed and relative speed

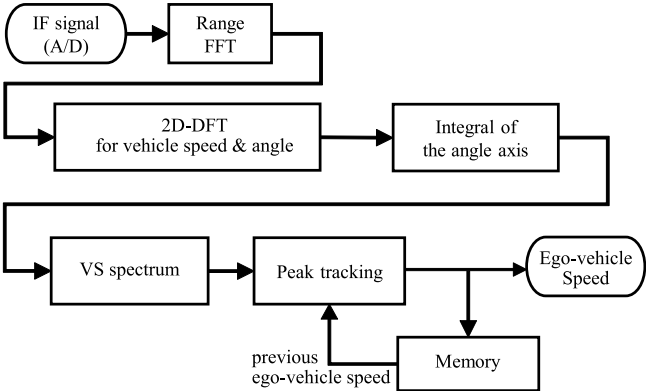

(b) flow chart

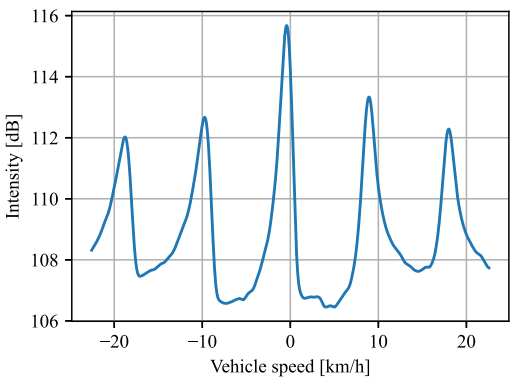

(c) vehicle speed spectrum

Fig. 1. Ego-vehicle speed estimation. 


\subsection{Estimation with vehicle speed spectrum}

The proposed method estimates the VS spectrum and estimates the ego-vehicle speed from the spectral peak. Figure 1(b) shows a flowchart of the method. The VS spectrum is estimated from the IF signal. IF signals are obtained $N$ times from an FCM radar with an $L$-element antenna and transformed to a complex range profile $R(k, n, l)$ using a fast Fourier transform (range-FFT). Here, $k=(0, \ldots, K-1)$, $n=(0, \ldots, N-1)$, and $l=(1, \ldots, L-1)$ are the range bin, IF signal number, and antenna number, respectively. The method estimates a 3D spectrum composed of the range, VS, and angle axes as follows:

$$
V(k, v, \theta)=\left|\sum_{l=0}^{L-1} \sum_{n=0}^{N-1} R(k, n . l) e^{j 2 \pi\left(\frac{n v \cos \theta}{2 v_{\text {max }}}+\frac{l \sin \theta}{2}\right)}\right|^{2},
$$

where $v$ is ego-vehicle speed, $v_{r_{\text {max }}}$ is maximum relative speed, and $\theta$ is the angle bin. When the vehicle travels at speed $v$, the peaks are distributed on the $v$-th plane on $V(k, v, \theta)$. A VS spectrum is estimated by integrating the $k$ and $\theta$ axes. Several peaks exist in the VS spectrum—see Fig. 1(c)—of which one represents the ego-vehicle speed; however, it is difficult to distinguish. Therefore, the proposed method tracks the peak near the previous ego-vehicle speed. The initial value is set to $0 \mathrm{~km} / \mathrm{h}$ and the speed is estimated using a high-resolution VS spectrum.

\section{Experiment}

An experiment was conducted using a measuring vehicle, and the specifications are shown in Fig. 2(a). A 79-GHz FCM radar module was equipped with a four-element antenna array for the receiver, and the angular resolution was $\sim 28.6^{\circ}$. The results of the method were compared with those of in-vehicle sensors. The wheel speed sensor and GNSS measured the VS and the distance traveled, respectively.

Four scenarios were defined, focusing on the speed, stop, and curves (Fig. 2(b)). Figure 2(c) shows the route in the scenarios. The vehicle traveled on a two-lane road on a Gunma University test course. The scenarios were based on Scenario A with cruising speed of $10 \mathrm{~km} / \mathrm{h}$. In Scenario B, the cruising speed was changed to $20 \mathrm{~km} / \mathrm{h}$. In Scenario C, the vehicle stopped before entering a crossing. In Scenario D, the vehicle traveled straight and then turned right. A driver checked the VS using a speedometer. We carried out Scenarios A-C thrice and Scenario D twice.

\section{Results}

\subsection{Vehicle speed}

Figures 3(a) and (b) show the results of the VS spectrum, which showed several clear peaks, and the estimation. The peak of the VS spectrum shifted from $0 \mathrm{~km} / \mathrm{h}$ to cruising speed after $10 \mathrm{~s}$ and to $0 \mathrm{~km} / \mathrm{h} 10 \mathrm{~s}$ before the measurement ended. In Scenario C, there was a peak shift at the stop.

The results for Scenarios A-C were close to the wheel speed. In Scenario D, the peak was divided when the vehicle turned right for $40 \mathrm{~s}$, and the results were lower than the wheel speed from 42 to $43 \mathrm{~s}$.

The results showed a faster start than the wheel speed. The difference was observed at the stop in Scenario C. 


\begin{tabular}{l|l}
\hline Contents & Parameter \\
\hline Sensor & Sharp TAKAYA 79 GHz FCM radar module \\
Frequency & $77.1-77.7 \mathrm{GHz}$ \\
Bandwidth & $600 \mathrm{MHz}$ \\
Antennas & Transmitted : 1 element \\
& Received : 4 elements \\
In-vehicle sensors & Wheel speed sensor \\
& GNSS \\
Vehicle & TOYOTA PULIUS \\
\hline
\end{tabular}

(a) specification sheet

\begin{tabular}{|c|c|c|c|}
\hline $\mathrm{A}$ & $\mathrm{B}$ & $\mathrm{C}$ & $\mathrm{D}$ \\
\hline \multicolumn{4}{|c|}{ Hold for $10 \mathrm{~s}$} \\
\hline \multirow{3}{*}{$\begin{array}{l}\text { Drive at } \\
10 \mathrm{~km} / \mathrm{h}\end{array}$} & \multirow{3}{*}{$\begin{array}{l}\text { Drive at } \\
20 \mathrm{~km} / \mathrm{h}\end{array}$} & $\begin{array}{l}\text { Drive at } \\
10 \mathrm{~km} / \mathrm{h}\end{array}$ & \multirow{2}{*}{$\begin{array}{l}\text { Drive at } \\
10 \mathrm{~km} / \mathrm{h}\end{array}$} \\
\hline & & $\begin{array}{l}\text { Stop at an } \\
\text { intersection }\end{array}$ & \\
\hline & & Drive & $\begin{array}{l}\text { Turn right at } \\
\text { curve }\end{array}$ \\
\hline \multicolumn{4}{|c|}{ Stop at the goal line } \\
\hline \multicolumn{4}{|c|}{ Hold for $10 \mathrm{~s}$} \\
\hline
\end{tabular}

(b) scenario table

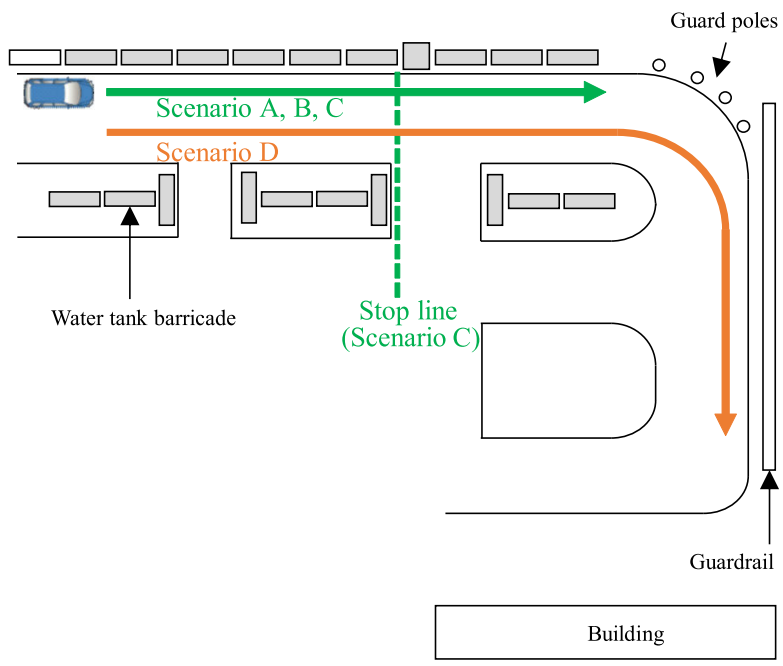

(c) experimental environment and routes

Fig. 2. Specifications of the experiment.

\subsection{Traveled distance}

The proposed method and the wheel speed sensor were compared in terms of the distance traveled, which was calculated by integrating the VS. Figure 3(c) shows the difference between the traveled distance of the estimated results and GNSS. "Difference" shows the difference between the proposed method and wheel speed. Compared with the GNSS, the traveled distance of the method was longer and the distance of the wheel sensor was shorter.

Differences were observed among the scenarios. In Scenario B, the mean difference of the proposed method was $\sim 0.5 \mathrm{~m}$ shorter than in Scenario A. In Scenario C, the mean difference in the wheel speed was $\sim 0.19 \mathrm{~m}$ shorter than that in Scenario A. In Scenario D, the difference between the method and wheel speed did not change by more than $0.2 \mathrm{~m}$ compared with Scenario A. The results between the first and second experiments in Scenario D differed owing to GNSS errors. 

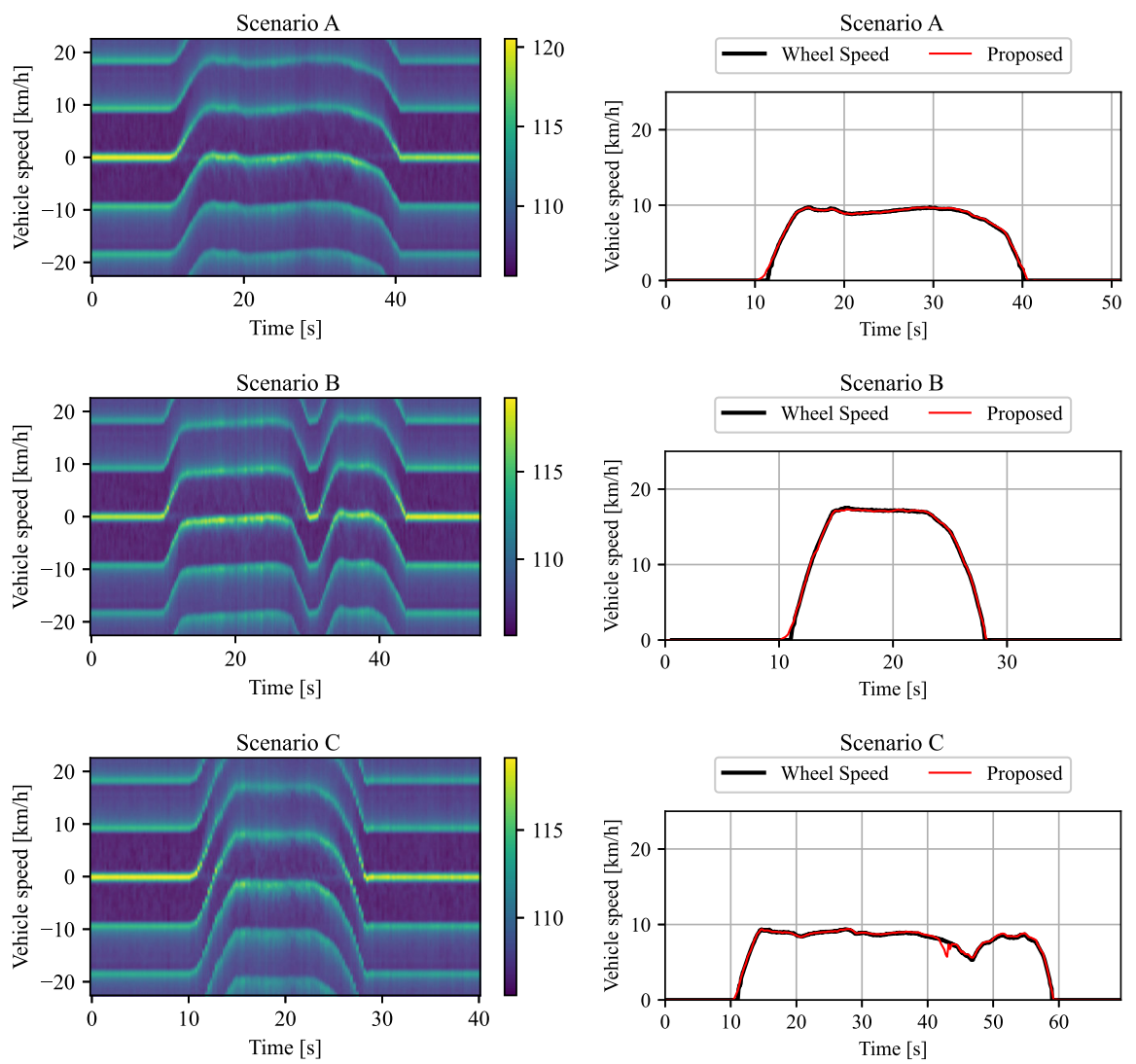

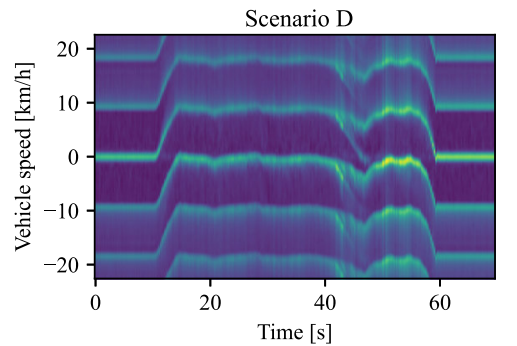

(a) vehicle speed spectrum

\begin{tabular}{|c|c|c|c|}
\hline \multirow{4}{*}{$\begin{array}{c}\text { Scenario } \\
\text { A }\end{array}$} & $\begin{array}{c}\text { Proposal } \\
{[\mathrm{m}]}\end{array}$ & $\begin{array}{l}\text { Wheel speed } \\
{[\mathrm{m}]}\end{array}$ & $\begin{array}{c}\text { Difference } \\
{[\mathrm{m}]}\end{array}$ \\
\hline & 0.42 & -0.42 & 0.84 \\
\hline & 0.43 & -0.35 & 0.78 \\
\hline & 0.31 & -0.43 & 0.74 \\
\hline \multirow{5}{*}{$\begin{array}{c}\text { Scenario } \\
\text { C }\end{array}$} & Proposal & Wheel speed & Difference \\
\hline & {$[\mathrm{m}]$} & {$[\mathrm{m}]$} & \\
\hline & 0.35 & -0.58 & 0.92 \\
\hline & 0.31 & -0.57 & 0.87 \\
\hline & 0.35 & -0.61 & 0.96 \\
\hline
\end{tabular}

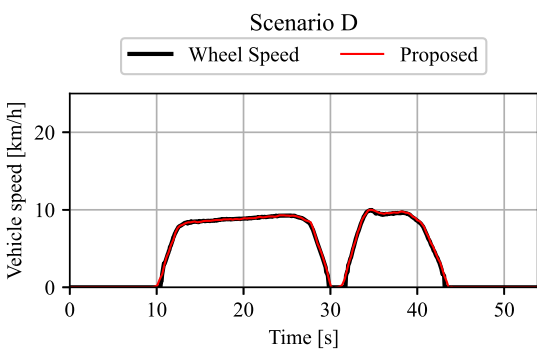

(b) vehicle speed

\begin{tabular}{|c|c|c|c|}
\hline \multirow{4}{*}{$\begin{array}{c}\text { Scenario } \\
\text { B }\end{array}$} & $\begin{array}{c}\text { Proposal } \\
{[\mathrm{m}]}\end{array}$ & $\begin{array}{c}\text { Wheel speed } \\
{[\mathrm{m}]}\end{array}$ & $\begin{array}{c}\text { Difference } \\
{[\mathrm{m}]}\end{array}$ \\
\hline & 0.03 & -0.32 & 0.35 \\
\hline & 0.00 & -0.25 & 0.25 \\
\hline & -0.04 & -0.22 & 0.18 \\
\hline \multirow{4}{*}{$\begin{array}{c}\text { Scenario } \\
\text { D }\end{array}$} & Proposal & Wheel speed & Difference \\
\hline & {$[\mathrm{m}]$} & {$[\mathrm{m}]$} & $\lceil\mathrm{m}\rceil$ \\
\hline & 0.73 & -0.18 & 0.92 \\
\hline & 0.31 & -0.54 & 0.85 \\
\hline
\end{tabular}

(c) traveled distance

Fig. 3. Results of the experiment.

\subsection{Discussion}

Slow speed estimation, the effect of cruising speed, and the effect of turning are discussed in this section.

The proposed method obtains a faster start as it can estimate a slow speed which cannot be measured by the wheel speed sensor. The error at the start caused the difference in wheel speed between Scenarios A and C; the travel distance in the 
proposed method did not differ between the scenarios.

The travel distance for the method was affected by the cruising speed. The distance traveled in Scenario B was shorter than that in the other scenarios because the accumulated error was smaller. In Scenario B, the travel time was $15 \mathrm{~s}$ shorter than in the other scenarios. If the mean VS error is $+0.1 \mathrm{~km} / \mathrm{h}$, the accumulated error becomes $+0.42 \mathrm{~m}$.

An error is observed in the curve of the proposed method. In Scenario D, the vehicle spectrum was divided, and the ego-vehicle speed was lower than the wheel speed when turning right. This is because the FCM radar did not face the travel direction owing to the yaw rate at the curve. When the vehicle turned, yaw rate $\phi$ occurred. The ego-vehicle speed is estimated as

$$
v=\frac{v_{r}}{\cos \theta}=\frac{v^{\prime} \cos (\theta-\phi)}{\cos \theta},
$$

where $v^{\prime}$ is the actual VS. The relative speed changes according to the yaw rate. The proposed method analyzes signals observed from surrounding objects, thus reducing the effect of a small yaw rate. The error lasted a second in Scenario D and was $<2 \mathrm{~km} / \mathrm{h}$. The accumulated error in Scenario D was estimated to be greater than that in Scenario A, but the traveled distance was not higher than that of Scenario A, owing to the error in ego-vehicle speed.

\section{Conclusion}

An ego-vehicle speed estimation based on spectral analysis using on-vehicle FCM radar was proposed. The proposed method estimates VS spectrum, and the egovehicle speed is subsequently estimated via peak tracking. An investigation using FCM radar with a four-element receiver antenna was conducted. The VS and traveled distance results obtained were compared with those of the wheel speed sensor and GNSS, which showed that the method can estimate low speeds that cannot be measured by the sensor. The method is applicable to low-angular-resolution FCM radar. When the vehicle turned, an error occurred because of the yaw rate. However, the difference between the distance of the method and GNSS was $<1 \mathrm{~m}$. Therefore, the method can effectively estimate ego-vehicle speed.

\section{Acknowledgments}

We would like to thank Editage [http://www.editage.jp] for editing and reviewing this manuscript for English language. 